\title{
Netrin-1/receptors regulate the pathogenesis in Parkinson's diseases
}

\author{
Eun Hee Ahn ${ }^{1,2}$, Seong Su Kang ${ }^{1}$, Keqiang Ye ${ }^{1}$ \\ 'Department of Pathology and Laboratory Medicine, Emory University School of Medicine, Atlanta, GA, USA \\ ${ }^{2}$ Department of Physiology, Hallym University College of Medicine, Chuncheon, Korea
}

Received: November 24, 2020

Revised: April 28, 2021

Accepted: April 30, 2021

Corresponding author:

Keqiang Ye

Department of Pathology and Laboratory Medicine, Emory

University School of Medicine, 141 Whitehead Bldg, 615

Michael St, Atlanta, GA 30322, USA

Tel: +1-404-712-2814

E-mail: kye@emory.edu
This is an Open Access article distributed under the terms of the Creative Commons Attribution Non-Commercial License (https:// creativecommons.org/licenses/ by-nc/4.0/).

\section{ABSTRACT}

The netrins and their receptors (deleted in colorectal cancer [DCC] and unc- 5 netrin receptors A-D [UNC5A-D]) play essential roles for central nervous system (CNS) development, mediating axonal and neuronal navigation. Emerging evidence indicates that they are implicated in maintaining the adult brain structure and mediating the pathogenesis of Parkinson's disease (PD). Recently, we reported the reduction of netrin-1 and brain-derived neurotrophic factor (BDNF) was inversely correlated with inflammatory cytokines-activated transcription factor CCAAT/enhancer binding protein $\beta$ (C/EBP $\beta$ ) in $\mathrm{PD}$ patient brains and colons. C/EBP $\beta$ binds to the promoters, repressing both netrin-1 and BDNF mRNA expression. Remarkably, netrin-1 deprivation triggered mammalian Ste20-like kinases 1 (MST1; Hippo) activation, which subsequently phosphorylated UNC5B and induced its apoptotic fragmentation via active caspase- 3 in dopaminergic neurons in the substantia nigra (SN). Moreover, deficiency of netrin-1 stimulated activation of delta-secretase (asparagine endopeptidase [AEP]) that cleaves $\alpha$-Syn at N103 and UNC5C receptor, facilitating PD pathologies. This review summarizes our current understanding of the crucial role of netrin-1 and its receptors in CNS and enteric nervous system contributing to PD pathologies by orchestrating multiple molecular players, including MST1, AEP, and C/EBP $\beta$. These findings support that blockade of AEP or stimulation of netrin-1 signaling may provide an innovative disease-modifying therapeutic strategy for treating PD.

Keywords: CCAAT-enhancer-binding proteins; DCC receptor; Delta-secretase; Netrins; Parkinson disease

\section{INTRODUCTION}

Netrin-1 is a secreted protein that belongs to class of laminin-related hormones involved in neuronal axon guidance [1,2]. Netrin-1 is genetically conserved across nematode worms [2], fruit flies, frogs, mice, and humans. The reported netrin-1 receptors are more than a dozen, including deleted in colorectal cancer (DCC) [3,4], unc-5 netrin receptor (UNC5) orthologues (human UNC5A-D and rodent UNC5H1-4) [5], P53 [6], several integrins [7,8], immunoglobulin superfamily of transmembrane receptors (neogenin) [9], p75 neurotrophin receptor (p75 NTR) 
[10], rearranged during transfection (RET) [11], anaplastic lymphoma kinase (ALK) [12], and tropomyosin receptor kinase (TrkC) [13]. They mediate a variety of biological functions from neuronal activity in adulthood [14], synaptic plasticity $[15,16]$ to inflammation $[17,18]$. DCC and UNC5H receptors are cleaved by major proteases of the apoptotic signal pathway, caspases, which are cysteine proteases that are activated at $\mathrm{pH} 7.4$, cleaving intracellular proteins on the carboxyl side of an aspartate residue. Caspase family members involved in apoptosis can be divided into two groups: signal cascade initiator caspases (caspase-8 and -9) and effector caspases (caspase-3, -6, and -7). Their activation initiates a proteolytic cascade leading to the activation of the effector caspases, rapidly triggering the execution of apoptosis. UNC5B and DCC receptors are cleaved by effector caspase (caspase-3) at specific amino acid position D412 for UNC5B and D1290 for DCC [3,5].

The midbrain structure is mainly composed of dopaminergic neurons which related the Parkinson's disease (PD) motor dysfunctions. Previously, it has been reported that the axons of dopaminergic neurons are guided by differential netrin-1 expression in the striatum (ST) via DCC receptor [19]. Also, netrin- 1 and DCC, UNC5B or UNC5C continue to be expressed in the adult brain, in the brain regions where neurodegenerative diseases like PD affected $[4,20]$. The preferential and progressive degeneration of dopaminergic neurons of the substantia nigra pars compacta (SNpc) region in PD leads to motor defect, resulting in impaired quality of life in patients with PD. However, netrin-1 and DCC are highly expressed in healthy (non-PD) dopaminergic neurons of the SNpc specifically $[21,22]$. Nowadays, the cause of neurodegenerative diseases remains obscure and the current therapies only temporarily alleviate the symptoms. Interestingly, netrin-1 is substantially reduced in the brain during aging and in the PD patient brains [23]. Of note, we found that netrin-1 deficiency activates mammalian Ste20-like kinases 1 (Mst1) that triggers UNC5B proteolytic fragmentation via T428 phosphorylation, making dopaminergic neurons particularly vulnerable to degeneration [23]. Moreover, we provide the molecular mechanism of how CCAAT/enhancer binding protein $\beta$ (C/EBP $\beta$ ) suppresses netrin-1 transcriptional expression in PD, contributing to its pathologies via activating asparagine endopeptidase (AEP). Remarkably, this protease also cleaves UNC5C receptor, exacerbating PD pathogenesis.

\section{NETRIN-1/RECEPTORS TUMORIGENESIS FUNCTIONS}

Mounting evidence supports that axon guidance molecules are implicated in various human cancers. The expression of netrin-1 is markedly reduced or deficient in approximately $50 \%$ of brain tumors and neuroblastomas [24] and in prostate cancer [25]. Some of the reports show that inactivation of netrin-1 contributes to cancer development. However, conflicting reports also demonstrate that netrin-1 acts as an oncogene in tumorigenesis. For instance, netrin-1 protein expression level is extremely high in colorectal cancer [26]. Accumulative evidence supports that netrin-1 inversely regulates DCC- and UNC5H receptors-mediated apoptosis and p53-dependent apoptosis. Mazelin et al. [27] generated transgenic (Tg) mice that overexpressed netrin- 1 in the gut. In this manuscript, s series of independent lines of Tg mice were obtained that had inserted a transgene containing netrin-1 complementary DNA under the gut-specific fatty acid-binding protein $(\mathrm{Fabpl})(4 \mathrm{x}$ at -132$)$ promoter18. Five of these lines (Tg-netrin-1/1 to 5) were analyzed. First of all, they shown netrin-1 (laminin-related secreted proteins, NTN1) expression of the transgene in the whole gastrointestinal (GI) tract. Moreover, tagged netrin-1 was detected by immunoblot and immunohistochemistry in epithelial villi and crypts isolated from the small intestine of Tg-netrin-1 mice. They address that inhibition of cell apoptosis by extrinsic increased expression of netrin-1 in mouse whole GI tract leads to the natural formation of hyperplastic and neoplastic lesions with these netrin- $1 \mathrm{Tg}$ mice. These results suggested that netrin-1 can promote colon cancer development, probably by regulating cell survival [27]. In addition to colorectal cancer [28,29], DCC deletion or mutation is also involved in testicular [30] and pancreatic cancers [31]. Hence, netrins and netrin receptors expression, gene deletion, and mutation are alternatively contributed to cancer's progression in the different organs. In addition to tumorigenesis, netrin-1 and its receptors are also implicated in the neurodegenerative diseases.

\section{MEDIATE PD PROGRESSION FROM THE ENTERIC NERVOUS SYSTEM TO THE CENTRAL NERVOUS SYSTEM VIA NETRIN-1, BRAIN-DERIVED NEUROTROPHIC FACTOR, AND C/EBP $\beta$ MOLECULES}

Hereafter, to explore the potential roles of netrin- 1 in sub- 
stantia nigra (SN) dopaminergic neurons, we analyzed its expression in the adult mammalian SN using Allen Human Brain Atlas data bank. In the database bank, netrin-1 expression in the adult brain is globally low except in some structures of the brainstem. Remarkably, netrin-1 expression level is the highest one among the genes in the SN. Moreover, NCBI Gene Expression Omnibus (GEO) database (GDS2821 and GDS 3129) shows the significant decrease of netrin-1 expression levels in PD patient's SN region; inversely, neuro-inflammation cytokines escalate transcription factor, C/EBP $\beta$ expression levels in overlapped brain region [32]. We have been identified the C/EBP $\beta$ transcription gene biological functions in neurodegenerative diseases [32-34].

The C/EBP $\beta$ acts brain-derived neurotrophic factor (BDNF)/ netrin-1 repressors upon neurotoxin and oxidative stress condition with interleukin 6 (IL-6), IL-1 1 , and tumor necrosis factor a cytokines overexpression. Moreover, C/EBP $\beta$ phosphorylation and activation are related the aging and neurodegenerative disease progression. Many research already identified C/EBP $\beta$ transcription factors in cytokines regulation. However, an unclear molecular mechanism remained in $\mathrm{PD}$. Therefore, we investigated the C/EBP $\beta$ possible molecular function with netrin-1/BDNF in PD to explore the unknown molecular mechanism.

Neurotrophins are mainly synthesized in central nervous system (CNS) as well as in enteric nervous system (ENS). Neurotrophins including BDNF were decreased in many different brain regions during the aging process [35-37]. BDNF is expressed in the hippocampus, frontal cortex, midbrain, amygdala, hypothalamus, ST, pons, and medulla oblongata $[38,39]$. Moreover, BDNF is also expressed in the non-neuronal peripheral cells such as $\mathrm{T}$ and $\mathrm{B}$ lymphocytes, monocytes, vascular endothelial, smooth, and skeletal muscle cells [4044]. BDNF promotes neuro-protection and neuro-regeneration. In PD animal models, BDNF promotes dopaminergic neuronal survival and improves neurotransmission and motor performance [45-47]. BDNF expression displays a similar pattern as netrin- 1 in human and rodent. They both are agedependent decreased in the brain [33].

Our most recent study reveals that C/EBP $\beta$ is progressively escalated in the brain, inducing AEP mRNA expression, which mediates Alzheimer's disease (AD) pathologies in animal models [34]. C/EBP $\beta$ belongs to the C/EBP family of transcription factors, binding to the CCAAT box of promoters and enhancer regions in numerous gene targets [48]. C/EBP $\beta$ is highly expressed in the intestine, liver, kidney, lungs, spleen, adipose tissue, pancreatic $\beta$-cells and in the CNS. C/EBP $\beta$ plays a key role in inflammation [49]. Promoters of many pro-inflammatory genes contain C/EBP $\beta$ consensus sequences [50]. Interestingly, the inflammation cytokines [34] and oxidative stress can induce $C / E B P \beta$ upregulation in dopaminergic neurons [51]. We found that both netrin-1 and BDNF were clearly diminished in PD patients' gut and brain tissue samples, inversely coupled with robust C/EBP $\beta$ (T235) phosphorylation, a marker for its activation [33]. Conceivably, C/EBP $\beta$ might act as a repressor to suppress both BDNF and netrin-1 mRNA transcriptional expression. To test this possibility, we identified that $\mathrm{C} / \mathrm{EBP} \beta$ bound to the promoters containing BDNF exon IV and netrin-1 exon I in specific binding sequence regions in primary neurons and neuroblastoma cells, repressing their mRNA expression (Fig. 1). To further test this notion, we employed 3 months old C/EBP $\beta$ heterozygous mice and wildtype (WT) littermates. After 2 months of rotenone oral gavages $(30 \mathrm{mg} / \mathrm{kg})$, both C/EBP $\beta$ and $\mathrm{p}-\mathrm{C} / \mathrm{EBP} \beta$ were evidently escalated in the gut and the brain of WT mice upon rotenone oral treatment. Accordingly, caspase-3 (apoptosis marker molecule) was activated, associated with UNC5B (netrin-1 receptor) and DCC (netrin-1 receptor) cleavage. Notably, tyrosine hydroxylase $(\mathrm{TH})$-positive dopaminergic neurons were evidently lost in the ENS in the gut, accompanied with extensive p-a-Syn S129 containing Lewy body-like inclusions. These events were diminished in $\mathrm{C} / \mathrm{EBP}^{+/-}$mice. Consequently, $\mathrm{C} /$ $\mathrm{EBP}^{+/-}$mice displayed mild GI tract dysfunction and damage as compared to the prominent effects in WT mice group [33].

$\mathrm{Gl}$ dysfunction is a prominent non-motor feature of PD. Before motor impairment, many PD patients suffer from delayed gastric emptying, constipation, and olfactory dysfunction [52-54]. Presumably, BDNF and netrin-1 reduction in the gut might account for the Gl tract aberrant pathological effects in PD. To test this notion, we generated BDNF or netrin-1 gut conditional knock out mice. Remarkably, these mice exhibited dopaminergic neuronal loss in the ENS in the gut, constipation, inflammation, and motor dysfunctions [33], which resemble to the PD prodromal symptoms. These interesting observations may shed light into the potential molecular mechanisms mediating PD patient GI tract dysfunctions. Furthermore, we have reported that AEP-cleaved a-Syn N103 and Tau N368 truncates associate with each other much stronger than their full-length counterparts. The pre-formed fibrils between $\alpha$-Syn N103 and Tau N368 spread from the gut along vagus nerve into the dorsal motor vagus nerves in the brainstem, from where they propagate into the SN, initiating PD pathologies and motor deficits [54]. Therefore, $C / E B P \beta$ activation that repressed both BDNF and ne- 
rat BDNF

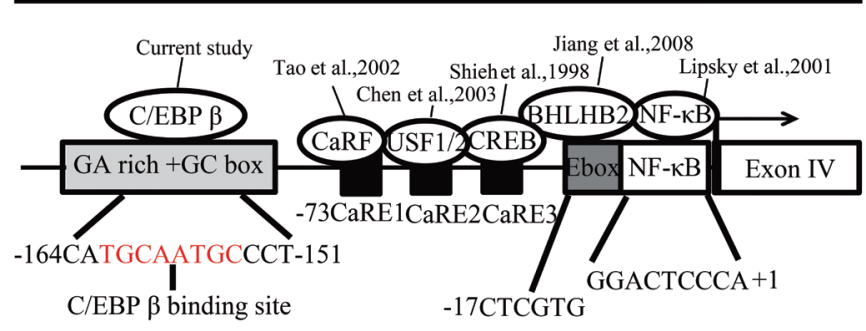

Human netrin-1

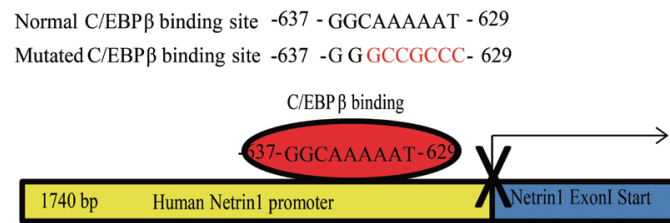

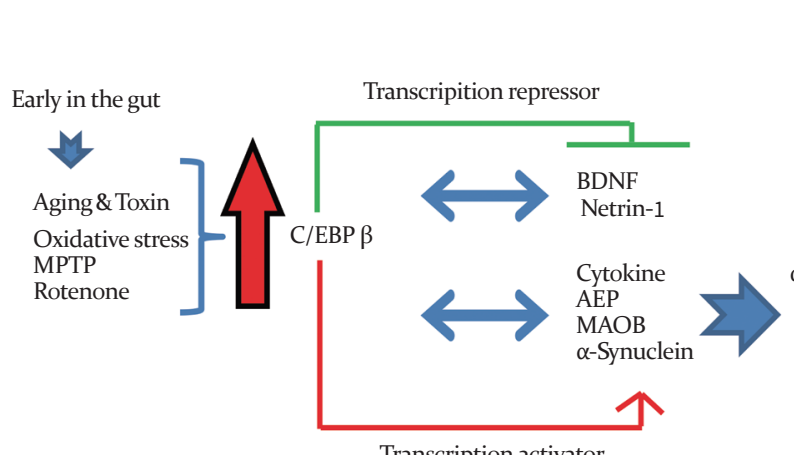

Transcription activator
Early in the gut

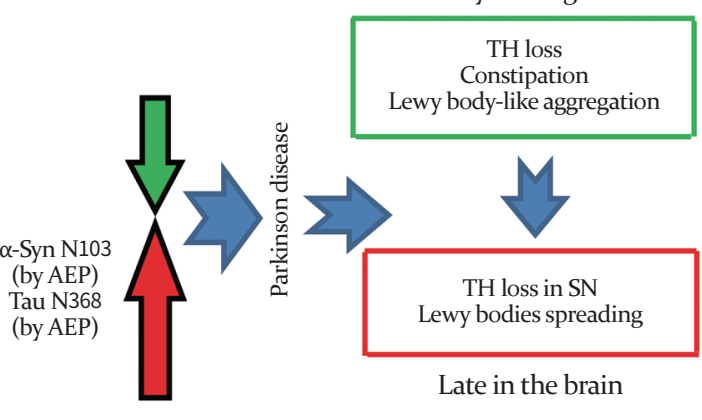

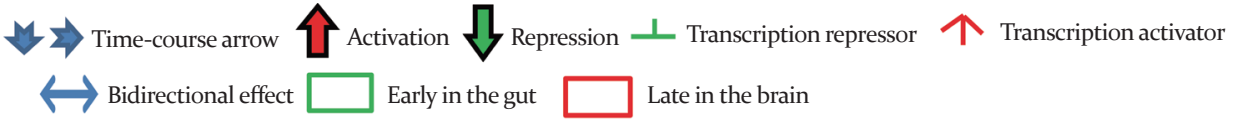

Fig. 1. CCAAT/enhancer binding protein $\beta(C / E B P \beta)$ binds brain-derived neurotrophic factor (BDNF) and netrin-1 promoter and acts as a transcriptional repressor. Schematic drawing of exon I-V with intron regions on rat BDNF gene. Rat BDNF gene promoter structure, and Exons are shown as boxes with different colors and introns are shown as lines (top, left). Human netrin-1 promoter gene schematic and C/ EBP $\beta$ and netrin-1 specific binding site diagram (top, right). A schematic model for C/EBP $\beta$, BDNF, and netrin-1 in Parkinson's disease pathogenesis molecular mechanism (bottom). GA, guanine adenine; GC, guanine cytosine; CaRF, calcium-responsive transcription factor; USF, upstream stimulatory factor; CREB, CAMP response element-binding protein; BHLHB, class E basic helix-loop-helix protein 40; NF-kB, nuclear factor-kB; MPTP, 1-methyl-4-phenyl-1,2,3,6-tetrahydropyridine; AEP, asparagine endopeptidase; MAOB, monoamine oxidase B; TH, tyrosine hydroxylase; SN, substantia nigra.

trin-1 expression levels in the gut, resulting in AEP activation and $\alpha$-Syn N103 and Tau N368 fragmentation, culminating in dopaminergic neuronal loss and prodromal GI disorders (Fig. 1). The truncated fragments form the Lewy body-like inclusions in the gut and they translocate into the brain, spreading the PD pathologies from the ENS into the CNS. Hence, $\mathrm{C} /$ EBP $\beta / A E P$ pathway provides an innovative molecular mechanism initiating $P D$ pathogenesis.

\section{NETRIN-1/DCC PAIR IN PD PATHOGENESIS VIA CASPASES}

The pair of netrin-1/DCC are key molecules in the CNS mediating axonal and neuronal guidance. The expression of netrin- 1 and DCC is maintained well in the adult brain; however, little is known about their roles in the mature neurons during the aging process. Interestingly, netrin-1 is highly expressed in the adult SN regions, which led us to investigate the role of the pair netrin-1/DCC in adult dopaminergic neuron's fate.

Especially, SNpc dopamine (DA) neurons are particularly susceptible to degeneration. Both in human and mouse midbrain, that ventral SNpc DA neurons exhibited a stronger signal for DCC than other midbrain DA neurons (from dorsal $\mathrm{SNpc}$ and the ventral tegmental area) $[21,55]$ suggesting that DCC or UNC 5 homologues receptors and netrin-1 protein levels may be markers for DA neurons that are most vulnerable to degeneration.

DCC and UNC5B, belong to the class of dependence receptors, a functional family of receptors shown to be cleaved and to trigger apoptosis in settings of poor receptor availability $[5,56]$. During the aging, the DCC and UNC-5 homologues levels were escalated and these receptors are truncated by active caspase-3. Mehlen et al. [3] discovered that DCC induces apoptosis in the absence of the netrin-1, but apoptosis is 
blocked when engaged by netrin-1. Hence, DCC receptor acts as a 'dependence receptor' [57,58]. Dependence receptors generate survival signaling on their specific ligands and induce apoptosis when uncoupled by the netrin-1 ligand. DCC was also shown to be a caspase substrate, cleaved at Asp1290 by caspase-3. A point mutation at this site suppresses the proapoptotic effect of DCC completely, indicating that DCC must be cleaved to induce apoptosis. Furthermore, a specific domain known as a dependence domain has been mapped to the intracellular region of DCC, which lies upstream of the caspase cleavage site. The deletion of this domain is strong enough to destroy the pro-apoptotic effect of DCC. Although the sequence of this domain is not related to a known death domain or a caspase involvement domain that allow protein-protein interactions between a number of apoptosis trigging proteins, it seems likely that the dependence domain functions as a start to recruit and activate caspase- 9 and caspase-3 [59]. DCC-induced apoptosis seems to be independent of both the mitochondrial apoptosis pathway and the death receptor/caspase-8 pathway. Instead, in the absence of netrin-1, DCC indirectly interacts with caspase- 9 through an unknown protein and stimulates the formation of the caspaseactivating complex, resulting in activation of caspase- 3 through caspase-9 without a requirement for the secrete of cytochrome c from the mitochondria or interaction with the scaffold protein apoptotic protease activating factor 1 (APAF1) [59].

Netrin-1/DCC pair tightly regulates cell survival and apoptosis. To explore netrin-1's pathological role in PD pathologies, we employed netrin-1 $\mathrm{fl} / \mathrm{fl}$ mice using Cre-virus injection into the SN region. We observed TH-positive neuronal loss and motor defects in netrin-1-depleted mice. A massive increase of terminal deoxynucleotidyl transferase dUTP nick end labeling (TUNEL) signals in TH-positive neurons in the SNpc demonstrated that conditional knockout of netrin-1 elicited death of dopaminergic neurons. Therefore, netrin-1 is the required element for DA neurons maintenance in adult animals. $\alpha$-Syn phosphorylation ( $p-\alpha-S y n$ S129), a PD pathological biomarker, and its aggregation were observed upon netrin-1 deprivation. The elevated DCC truncate and active caspase- 3 were identified in the PD animal model. In primary neuronal cultures, neutralization of netrin-1 $[60,61]$ escalated DCC or UNC5B protein levels and cleavage, associated with increased neuronal apoptosis. On the other hand, DCC or UNC5B expression levels triggered caspase's activation in dopaminergic neurons. However, DCC might be a doubleedged sword for dopaminergic neurons depending on netrin-1 protein availability: supporting neuronal survival (and outgrowth) in the presence of netrin-1 and promoting cell death in conditions of trophic scarcity. As dopaminergic neurons are thought to be vulnerable to oxidative stress and inflammation due to their unique morphology and mitochondrial dysfunctions, DCC dual signaling could be a neuronal cell protective mechanism, eliminating damaged or disable neurons and protecting healthy neurons from additional harm. Our current research showed that netrin-1 silencing/ deprivation were associated with increased $\alpha$-Syn, monoamine oxidase-B (MAOB), C/EBP $\beta$, DCC, UNC5B, caspase-3, a -Syn pS129, aggregates $\alpha$-Syn, and AEP levels [33,62]. Moreover, netrin-1 expression and protein levels were progressively reduced in an age-dependent manner in the adult SN of alpha-synuclein gene (SNCA) Tg mice, emphasizing that there might be a link between $\alpha$-Syn and netrin-1 levels. Injection of recombinant netrin-1 prevented behavioral motor dysfunctions in SNCA Tg mice with the reduced cleaved form of DCC, UNC5B during the caspase-3 and AEP enzyme activation $[33,62]$.

\section{NETRIN-1 DEPRIVATION ACTIVATES MST1 THAT PHOSPHORYLATES UNC5B, PROMOTING CELL DEATH}

The MST1/2 (homologs of Drosophila Hippo) phosphorylates and activates large tumor suppressor $1 / 2$ (LATS1/2) [63]. The Hippo pathway in both Drosophila and mammals regulates cell number by modulating cell death, cell proliferation, and cell differentiation. Orchestration of these processes plays a critical role in a variety of physiological and pathological conditions [64]. During development, an increase in cell number is an essential requirement for supporting organ maturation and functions; meanwhile, modest differentiation of multiple cell types warrants the appropriate functions of developed organs. In several studies, the Hippo (Mst1) pathway has been shown to induce cell death and differentiation. MST1/2 possesses additional functions in addition to regulating Hippo pathway components of LATS1/2 and yes-associated protein (YAP)/transcriptional coactivator with PDZbinding motif (TAZ). YAP and TAZ are transcriptional co-activators. When translocated into the nucleus, they regulate gene expression through interaction with TEA domain transcription factor 1-4, which are sequence-specific transcription factors that mediate the main transcriptional output of the Hippo pathway in mammalian cells [65]. The major physiological functions of YAP and TAZ are to induce cell survival and proliferation [66]. 
MST1 pathway is implicated in neuronal cell death. For example, Mst1/2 phosphorylates forkhead box protein 01 (FOXO1) to elicit its nuclear localization and transcription of genes promoting apoptosis in mammalian neurons [67]. Moreover, reduced Mst1 phosphorylation eliminates blood-brain barrier (BBB) damage, and neurobehavioral impairment and brain edema [68]. Previously, we showed that Akt phosphorylates MST1 on T387 and inhibits its proteolytic activation, blocking FOXO3 phosphorylation and nuclear translocation and stimulating cell survival [69]. Further, we have reported that netrin-1 induces the interaction of UNC5B receptor with the brain-specific GTPase phosphoinositide-3 kinase enhancer $\mathrm{L}$ (PIKE-L) [70]. This interaction stimulates PI3K/Akt activation, inhibits UNC5B receptor pro-apoptotic activity and enhances neuronal cell survival [71]. In our recent study, we reported that netrin-1 deprivation mediates MST1 activation in UNC5B receptor-dependent manner, and active Mst1 phosphorylates UNC5B, promoting its apoptotic cleavage through caspase-3 activation. Netrin-1 reduction stimulates dopaminergic neuronal loss in PD patient brain samples or mouse brain samples via activating MST1 that subsequently phosphorylates UNC5B on T428 residue, escalating its proteolytic cleavage and apoptosis [23]. Overexpression of un-phosphorylate UNC5B T428A mutant strongly suppressed netrin-1 deprivation-induced MST1 apoptotic activation, leading to repression of $p$-LATS/p-YAP pathway, which culminates in repression of caspase-3 activation and inhibition of dopaminergic apoptosis. Blockade of UNC5B T428 phosphorylation by MST1 not only inhibits UNC5B apoptotic cleavage and reduces its apoptotic activity but also diminishes MST1 apoptotic activation (Fig. 2). Hence, these findings support that UNC5B and MST1 mutually regulate each other apoptotic functions via physical interaction and phosphorylation, and netrin-1 modulates Hippo/MST1 signaling via its receptor UNC5B receptor [23].

MST1/2 are also involved in cellular oxidative stress responses $[67,72]$. On the other hand, YAP physically interacts with FOXO1 and activates FOXO1-mediated transcription of catalase and manganese superoxide dismutase (MnSOD) genes and thereafter reduces levels of reactive oxygen species (ROS) and ischemia/reperfusion-induced injury in the heart [73], suggesting a physiological role of YAP in ROS scavenge. Noticeably, MST1 is activated in neurodegenerative prion disease and neuro-inflammation mechanism [74]. Moreover, YAP has also been implicated in neurodegenerative diseases, for instance, Huntington's disease. In transcriptional repression-induced atypical death of neurons, YAP full-length is reduced, triggering apoptosis [75]. Our recent studies shown that netrin-1 induces YAP levels via inhibiting Hippo/MST1 pathway and promotes cell survival are in alignment with the well-defined pro-survival functions of YAP $[23,76]$.

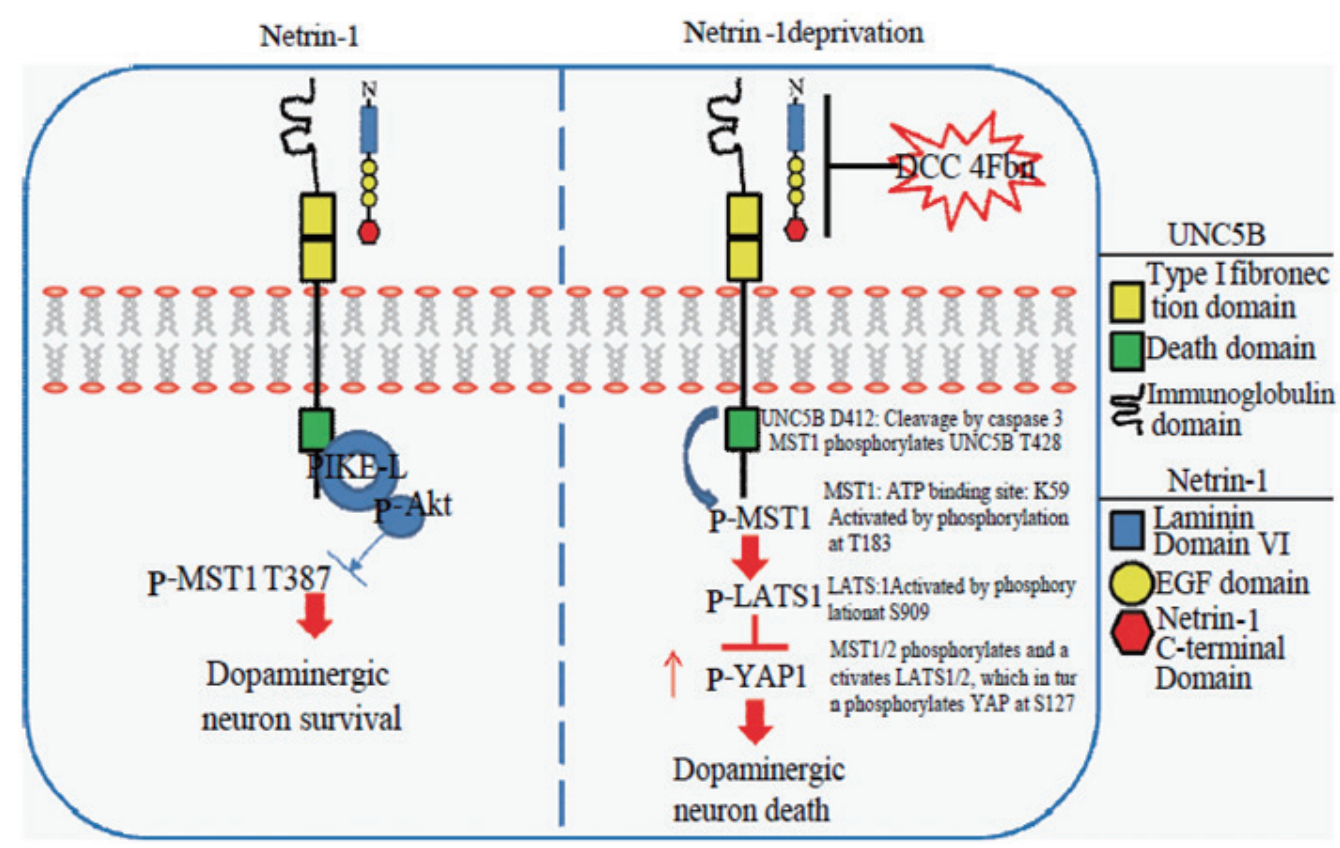

Fig. 2. The schematic model of netrin-1 deprivation-elicited Hippo/mammalian Ste20-like kinases 1 (MST1) signaling pathway via unc-5 netrin receptor B (UNC5B). PIKE-L, phosphoinositide-3 kinase enhancer L; DCC, deleted in colorectal cancer; ATP, adenosine triphosphate; LATS1/2, large tumor suppressor 1/2; YAP1, yes-associated protein 1; EGF, epidermal growth factor. 


\section{NETRIN-1 DEFICIENCY INDUCES AEP ACTIVATION AND UNC5C CLEAVAGE IN PD PATHOGENESIS}

Netrin-1 receptors including DCC and UNC5Hs act as dependence receptors regulating neuronal apoptosis. Recently, we reported that AEP cleaves human $\alpha$-Syn, promotes its aggregation and escalates its neurotoxicity, thus leading to dopaminergic neuronal death and motor dysfunctions in PD mouse model. AEP enzyme is activated and consequently cleaves human $\alpha$-Syn at $\mathrm{N} 103$ residue in an age-dependent manner in SNCA Tg mice, and AEP is highly active in the SNpc regions in human brains with PD. Deletion of AEP from SNCA Tg mice alleviates dopaminergic neuronal loss and attenuates motor deficits [77]. Previous studies show that UNC5C expression is down-regulated in colorectal cancers and in several other cancers. This is also the case for UNC5A and UNC5B, mainly through promoter methylation [78-80]. Nevertheless, the tumor frequency is not increased in UN5C-deficient mice, suggesting that loss of UNC5C function is not sufficient to initiate tumorigenesis in mice. UNC5C serves as receptor in signaling pathways, recruiting other proteins into signaling complexes $[81,82]$. UNC5C is also widely expressed in the adult CNS neurons. It plays a critical role in the development of spinal accessory motor neurons [83]. Moreover, UNC5C functions as a chemotropic molecule in mediating axon growth and neuronal transplantation in neuronal development [84-87]. So far, six single nucleotide polymorphisms in UNC5C have been show to increase the risk of late-onset $A D$ [88]. A recent mechanistic study showed that UNC5C T835M mutant in death domain induced neuronal cell apoptosis via serine/threonine death-associated protein kinase (DAPK1)/protein kinase D (PKD)/apoptosis signal-regulating kinase 1 (ASK1)/nicotinamide adenine dinucleotide phosphate (NADPH) oxidase/ caspases pathways [20]. In our most recent unpublished work, we show that netrin-1 reduction in PD patient brains or induced pluripotent stem cell (iPSC)-induced human neurons induces AEP activity, which robustly cleaves UNC5C at both $\mathrm{N} 467$ and N547 residues, augmenting TH-positive dopaminergic neuronal cell apoptosis. Moreover, we identified the netrin-1 is age-dependently repressed in the brains of SNCA Tg mice, associated with AEP activation and UNC5C fragmentation and dopaminergic neuronal loss. In alignment with these results, we observed that human $\alpha$-Syn N103 truncation by AEP is highly escalated in PD brains and aged human SNCA Tg mouse brains, correlating with elevated p- $\alpha$-Syn S129 activities. Consistently, overexpression of AEP-truncated UNC5C C-terminal fragment in the SN of human SNCA Tg mice strongly

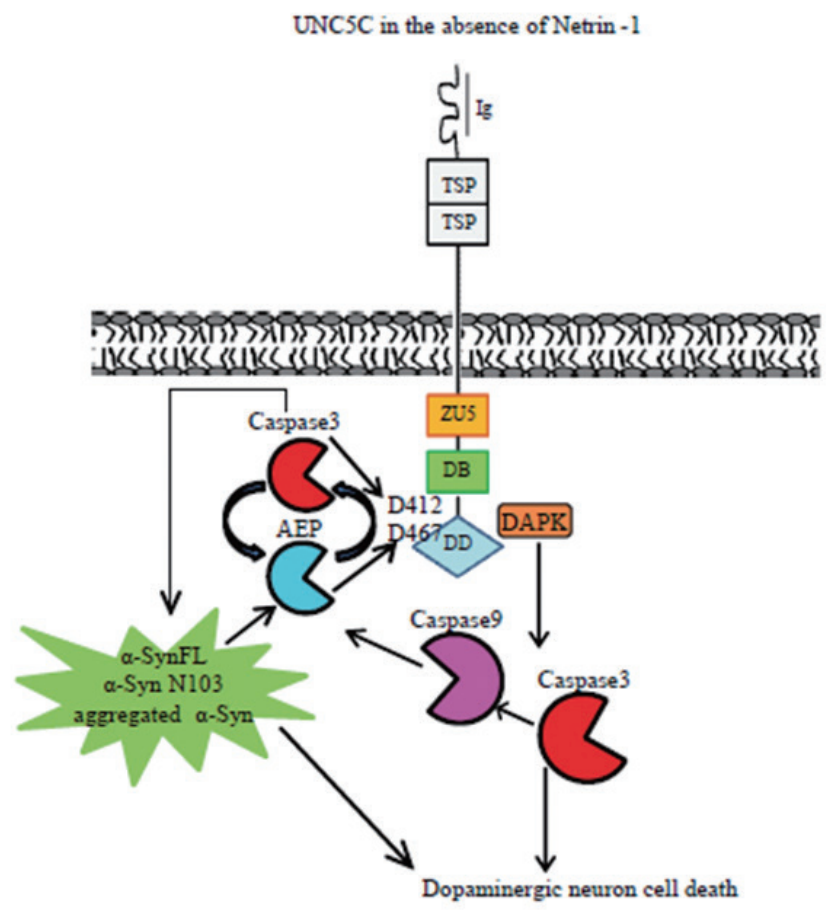

Fig. 3. The netrin-1 reduction is associated with asparagine endopeptidase (AEP) activation and unc-5 netrin receptor C (UNC5C) cleavage. A schematic model for UNC5C, AEP, and caspase-3 in Parkinson's disease pathogenesis molecular mechanism in the absence of netrin-1. Ig, immunoglobulin; TSP, thrombospondin type 1; ZU5, zona occludins 5 domain; DB, DCC-binding domain; DD, death domain; DAPK, serine/ threonine death-associated protein kinase; SynFL, sunuclein full length. 
augments caspase- 3 and AEP activation, accompanied with conspicuous dopaminergic neuronal cell loss, leading to $\alpha$-Syn aggregation and motor dysfunctions. Therefore, netrin-1 deprivation in PD might induce both caspase- 3 and AEP activation, which robustly cleave DCC and UNC5C that subsequently trigger cell death signals, culminating in dopaminergic neuronal cell apoptosis and defects (Fig. 3).

\section{CONCLUSION}

We have summarized the evidence that DCC, UNC5H family receptors, AEP, and C/EBP $\beta$ are crucial neuronal cell death factors in PD. Netrin-1 and BDNF are strongly reduced in the PD patient brains and gut tissues and rotenone-treated mice, and they also decline in SNCA Tg mice in an age-dependent manner. These innovative discoveries strongly support that netrin-1 and its receptors are potential targets for drug development for PD. Netrin-1 is mainly expressed in the brainstem and notably in the SN. These structures are predominantly affected by Lewy body pathology and neurodegeneration in $P D$. Netrin-1 deficiency triggers AEP upregulation via activating C/EBP $\beta$ transcription factor. In addition, $C / E B P \beta$ also acts as a repressor for both BDNF and netrin-1 via binding to their promoters [33]. C/EBP $\beta$ has different binding partners under different environmental conditions. C/EBP $\beta$ directly regulates IL-6, IL-1 $\beta$, and nuclear factor-kB transcription under oxidative stress condition [49]. DA metabolite by MAOB, 3,4-dihydroxyphenylacetaldehyde (DOPAL), strongly induces AEP enzyme activity, whose mRNA transcription mediated by $\mathrm{C} /$ EBP $\beta$ in the CNS $[34,89]$. Hence, our results support the notion that alleviating netrin-1 reduction via preventing neuroinflammation or oxidative stress-activated C/EBP $\beta / A E P$ repression might be the potential therapeutic strategy.

\section{CONFLICTS OF INTEREST}

No potential conflict of interest relevant to this article was reported. Keqiang Ye has been on the editorial board of P\&FM since March 2017. He was not involved in the review process of this article.

\section{ACKNOWLEDGMENTS}

This work was supported by grants from National Institutes of Health (RF1 AG051538; RO1 AG065517) to Keqiang Ye. We thank Dr. Arthur W. English at the Department of Cell Biology for proofreading the manuscript.

\section{ORCID}

Eun Hee Ahn https://orcid.org/0000-0002-1833-6720

Seong Su Kang https://orcid.org/0000-0002-2517-9962

Keqiang Ye https://orcid.org/0000-0002-7657-8154

\section{AUTHOR CONTRIBUTIONS}

Conception or design: EHA, KY.

Acquisition, analysis, or interpretation of data: EHA, KY.

Drafting the work or revising: EHA, SSK, KY.

Final approval of the manuscript: EHA, SSK, KY.

\section{REFERENCES}

1. Kennedy TE, Serafini T, de la Torre JR, Tessier-Lavigne M. Netrins are diffusible chemotropic factors for commissural axons in the embryonic spinal cord. Cell 1994;78:42535.

2. Serafini T, Kennedy TE, Galko MJ, Mirzayan C, Jessell TM, Tessier-Lavigne $M$. The netrins define a family of axon outgrowth-promoting proteins homologous to C. elegans UNC-6. Cell 1994;78:409-24.

3. Mehlen P, Rabizadeh S, Snipas SJ, Assa-Munt N, Salvesen GS, Bredesen DE. The DCC gene product induces apoptosis by a mechanism requiring receptor proteolysis. $\mathrm{Na}$ ture 1998;395:801-4.

4. Livesey FJ, Hunt SP. Netrin and netrin receptor expression in the embryonic mammalian nervous system suggests roles in retinal, striatal, nigral, and cerebellar development. Mol Cell Neurosci 1997;8:417-29.

5. Llambi F, Causeret F, Bloch-Gallego E, Mehlen P. Netrin-1 acts as a survival factor via its receptors UNC5H and DCC. EMBO J 2001;20:2715-22.

6. Arakawa H. p53, apoptosis and axon-guidance molecules. Cell Death Differ 2005;12:1057-65.

7. Yebra M, Montgomery AM, Diaferia GR, Kaido T, Silletti S, Perez B, et al. Recognition of the neural chemoattractant Netrin-1 by integrins alpha6beta 4 and alpha3beta1 regulates epithelial cell adhesion and migration. Dev Cell 2003; 5:695-707.

8. Stupack DG, Puente XS, Boutsaboualoy S, Storgard CM, Cheresh DA. Apoptosis of adherent cells by recruitment of caspase-8 to unligated integrins. J Cell Biol 2001;155: 459-70.

9. Matsunaga E, Tauszig-Delamasure S, Monnier PP, Mueller 
PRECISION AND FUTURE MIEDICINE

Netrin's function in neurodegenerative diseases

BK, Strittmatter SM, Mehlen P, et al. RGM and its receptor neogenin regulate neuronal survival. Nat Cell Biol 2004;6: 749-55.

10. Rabizadeh S, Oh J, Zhong LT, Yang J, Bitler CM, Butcher LL, et al. Induction of apoptosis by the low-affinity NGF receptor. Science 1993;261:345-8.

11. Bordeaux MC, Forcet C, Granger L, Corset V, Bidaud C, Billaud $\mathrm{M}$, et al. The RET proto-oncogene induces apoptosis: a novel mechanism for Hirschsprung disease. EMBO J 2000;19:4056-63.

12. Mourali J, Benard A, Lourenco FC, Monnet C, Greenland C, Moog-Lutz C, et al. Anaplastic lymphoma kinase is a dependence receptor whose proapoptotic functions are activated by caspase cleavage. Mol Cell Biol 2006;26:620922.

13. Tauszig-Delamasure S, Yu LY, Cabrera JR, Bouzas-Rodriguez J, Mermet-Bouvier C, Guix C, et al. The TrkC receptor induces apoptosis when the dependence receptor notion meets the neurotrophin paradigm. Proc Natl Acad Sci U S A 2007;104:13361-6.

14. Lai Wing Sun K, Correia JP, Kennedy TE. Netrins: versatile extracellular cues with diverse functions. Development 2011;138:2153-69.

15. Bayat M, Baluchnejadmojarad T, Roghani M, Goshadrou F, Ronaghi A, Mehdizadeh M. Netrin-1 improves spatial memory and synaptic plasticity impairment following global ischemia in the rat. Brain Res 2012;1452:185-94.

16. Horn KE, Glasgow SD, Gobert D, Bull SJ, Luk T, Girgis J, et al. DCC expression by neurons regulates synaptic plasticity in the adult brain. Cell Rep 2013;3:173-85.

17. Podjaski C, Alvarez JI, Bourbonniere L, Larouche S, Terouz S, Bin JM, et al. Netrin 1 regulates blood-brain barrier function and neuroinflammation. Brain 2015;138(Pt 6): 1598-612.

18. He X, Liu Y, Lin X, Yuan F, Long D, Zhang Z, et al. Netrin-1 attenuates brain injury after middle cerebral artery occlusion via downregulation of astrocyte activation in mice. J Neuroinflammation 2018;15:268.

19. Li J, Duarte T, Kocabas A, Works M, McConnell SK, Hynes MA. Evidence for topographic guidance of dopaminergic axons by differential Netrin-1 expression in the striatum. Mol Cell Neurosci 2014;61:85-96.

20. Hashimoto Y, Toyama Y, Kusakari S, Nawa M, Matsuoka M. An Alzheimer disease-linked rare mutation potentiates netrin receptor uncoordinated-5C-induced signaling that merges with amyloid $\beta$ precursor protein signaling. J Biol Chem 2016;291:12282-93.
21. Osborne PB, Halliday GM, Cooper HM, Keast JR. Localization of immunoreactivity for deleted in colorectal cancer (DCC), the receptor for the guidance factor netrin-1, in ventral tier dopamine projection pathways in adult rodents. Neuroscience 2005;131:671-81.

22. Penttinen AM, Suleymanova I, Albert K, Anttila J, Voutilainen MH, Airavaara M. Characterization of a new lowdose 6-hydroxydopamine model of Parkinson's disease in rat. J Neurosci Res 2016;94:318-28.

23. Ahn EH, Kang SS, Qi Q, Liu X, Ye K. Netrin1 deficiency activates MST1 via UNC5B receptor, promoting dopaminergic apoptosis in Parkinson's disease. Proc Natl Acad Sci U S A 2020;117:24503-13.

24. Meyerhardt JA, Caca K, Eckstrand BC, Hu G, Lengauer C, Banavali S, et al. Netrin-1: interaction with deleted in colorectal cancer (DCC) and alterations in brain tumors and neuroblastomas. Cell Growth Differ 1999;10:35-42.

25. Latil A, Chene L, Cochant-Priollet B, Mangin P, Fournier G, Berthon P, et al. Quantification of expression of netrins, slits and their receptors in human prostate tumors. Int J Cancer 2003;103:306-15.

26. Mehlen P, Llambi F. Role of netrin-1 and netrin-1 dependence receptors in colorectal cancers. $\mathrm{Br}$ J Cancer 2005; 93:1-6.

27. Mazelin L, Bernet A, Bonod-Bidaud C, Pays L, Arnaud S, Gespach C, et al. Netrin-1 controls colorectal tumorigenesis by regulating apoptosis. Nature 2004;431:80-4.

28. Fearon ER, Cho KR, Nigro JM, Kern SE, Simons JW, Ruppert JM, et al. Identification of a chromosome $18 \mathrm{q}$ gene that is altered in colorectal cancers. Science 1990;247:4956.

29. Thiagalingam S, Lengauer C, Leach FS, Schutte M, Hahn SA, Overhauser J, et al. Evaluation of candidate tumour suppressor genes on chromosome 18 in colorectal cancers. Nat Genet 1996;13:343-6.

30. Murty VV, Li RG, Houldsworth J, Bronson DL, Reuter VE, Bosl GJ, et al. Frequent allelic deletions and loss of expression characterize the DCC gene in male germ cell tumors. Oncogene 1994;9:3227-31.

31. Hilgers W, Song JJ, Haye M, Hruban RR, Kern SE, Fearon ER. Homozygous deletions inactivate DCC, but not MADH4/ DPC4/SMAD4, in a subset of pancreatic and biliary cancers. Genes Chromosomes Cancer 2000;27:353-7.

32. Wang ZH, Xiang J, Liu X, Yu SP, Manfredsson FP, Sandoval IM, et al. Deficiency in BDNF/TrkB neurotrophic activity stimulates $\delta$-secretase by upregulating C/EBP $\beta$ in Alzheimer's disease. Cell Rep 2019;28:655-69. 
33. Ahn EH, Kang SS, Liu X, Cao X, Choi SY, Musazzi L, et al. $B D N F$ and netrin- 1 repression by $C / E B P \beta$ in the gut triggers Parkinson's disease pathologies, associated with constipation and motor dysfunctions. Prog Neurobiol 2021;198: 101905.

34. Wang ZH, Gong K, Liu X, Zhang Z, Sun X, Wei ZZ, et al. C/ EBP $\beta$ regulates delta-secretase expression and mediates pathogenesis in mouse models of Alzheimer's disease. Nat Commun 2018;9:1784.

35. Bishop NA, Lu T, Yankner BA. Neural mechanisms of ageing and cognitive decline. Nature 2010;464:529-35.

36. Samanez-Larkin GR, Knutson B. Decision making in the ageing brain: changes in affective and motivational circuits. Nat Rev Neurosci 2015;16:278-89.

37. Huang EJ, Reichardt LF. Neurotrophins: roles in neuronal development and function. Annu Rev Neurosci 2001;24: 677-736.

38. Murer MG, Yan Q, Raisman-Vozari R. Brain-derived neurotrophic factor in the control human brain, and in Alzheimer's disease and Parkinson's disease. Prog Neurobiol 2001;63:71-124.

39. Tapia-Arancibia L, Aliaga E, Silhol M, Arancibia S. New insights into brain BDNF function in normal aging and Alzheimer disease. Brain Res Rev 2008;59:201-20.

40. Conner JM, Lauterborn JC, Yan Q, Gall CM, Varon S. Distribution of brain-derived neurotrophic factor (BDNF) protein and mRNA in the normal adult rat CNS: evidence for anterograde axonal transport. J Neurosci 1997;17:2295313.

41. Kerschensteiner M, Gallmeier E, Behrens L, Leal W, Misgeld T, Klinkert WE, et al. Activated human T cells, B cells, and monocytes produce brain-derived neurotrophic factor in vitro and in inflammatory brain lesions: a neuroprotective role of inflammation? J Exp Med 1999;189: 865-70.

42. Nakahashi T, Fujimura H, Altar CA, Li J, Kambayashi J, Tandon NN, et al. Vascular endothelial cells synthesize and secrete brain-derived neurotrophic factor. FEBS Lett 2000;470:113-7.

43. Donovan MJ, Miranda RC, Kraemer R, McCaffrey TA, Tessarollo L, Mahadeo D, et al. Neurotrophin and neurotrophin receptors in vascular smooth muscle cells. Regulation of expression in response to injury. Am J Pathol 1995; 147:309-24.

44. Yarrow JF, White LJ, McCoy SC, Borst SE. Training augments resistance exercise induced elevation of circulating brain derived neurotrophic factor (BDNF). Neurosci
Lett 2010;479:161-5.

45. Zuccato C, Cattaneo E. Brain-derived neurotrophic factor in neurodegenerative diseases. Nat Rev Neurol 2009;5: 311-22.

46. Autry AE, Monteggia LM. Brain-derived neurotrophic factor and neuropsychiatric disorders. Pharmacol Rev 2012; 64:238-58.

47. Allen SJ, Watson JJ, Shoemark DK, Barua NU, Patel NK. GDNF, NGF and BDNF as therapeutic options for neurodegeneration. Pharmacol Ther 2013;138:155-75.

48. van der Krieken SE, Popeijus HE, Mensink RP, Plat J. CCAAT/enhancer binding protein $\beta$ in relation to ER stress, inflammation, and metabolic disturbances. Biomed Res Int 2015;2015:324815.

49. Poli V. The role of C/EBP isoforms in the control of inflammatory and native immunity functions. J Biol Chem 1998; 273:29279-82.

50. Caivano M, Gorgoni B, Cohen P, Poli V. The induction of cyclooxygenase- 2 mRNA in macrophages is biphasic and requires both CCAAT enhancer-binding protein beta (C) EBP beta) and C/EBP delta transcription factors. J Biol Chem 2001;276:48693-701.

51. Wu Z, Xia Y, Wang Z, Su Kang S, Lei K, Liu X, et al. C/EBPß/ $\delta$-secretase signaling mediates Parkinson's disease pathogenesis via regulating transcription and proteolytic cleavage of $\alpha$-synuclein and MAOB. Mol Psychiatry 2021;26: 568-585.

52. Abbott RD, Petrovitch H, White LR, Masaki KH, Tanner CM, Curb JD, et al. Frequency of bowel movements and the future risk of Parkinson's disease. Neurology 2001;57:45662.

53. Doty RL, Deems DA, Stellar S. Olfactory dysfunction in parkinsonism: a general deficit unrelated to neurologic signs, disease stage, or disease duration. Neurology 1988 38:1237-44.

54. Ahn EH, Kang SS, Liu X, Chen G, Zhang Z, Chandrasekharan $B$, et al. Initiation of Parkinson's disease from gut to brain by $\delta$-secretase. Cell Res 2020;30:70-87.

55. Reyes S, Fu Y, Double KL, Cottam V, Thompson LH, Kirik D, et al. Trophic factors differentiate dopamine neurons vulnerable to Parkinson's disease. Neurobiol Aging 2013;34: 873-86.

56. Yetnikoff L, Labelle-Dumais C, Flores C. Regulation of netrin-1 receptors by amphetamine in the adult brain. Neuroscience 2007;150:764-73.

57. Braekevelt CR. Retinal photoreceptor fine structure in the red-backed salamander (Plethodon cinereus). Histol His- 
PRECISION AND FUTURE MIEDICINE

Netrin's function in neurodegenerative diseases

topathol 1992;7:463-70.

58. Mehlen P, Bredesen DE. The dependence receptor hypothesis. Apoptosis 2004;9:37-49.

59. Forcet C, Ye X, Granger L, Corset V, Shin H, Bredesen DE, et al. The dependence receptor DCC (deleted in colorectal cancer) defines an alternative mechanism for caspase activation. Proc Natl Acad Sci U S A 2001;98:3416-21.

60. Broutier L, Creveaux M, Vial J, Tortereau A, Delcros JG, Chazot G, et al. Targeting netrin-1/DCC interaction in diffuse large B-cell and mantle cell lymphomas. EMBO Mol Med 2016;8:96-104.

61. Paradisi A, Maisse C, Coissieux MM, Gadot N, Lepinasse F, Delloye-Bourgeois $C$, et al. Netrin-1 up-regulation in inflammatory bowel diseases is required for colorectal cancer progression. Proc Natl Acad Sci U S A 2009;106:17146-51.

62. Jasmin M, Ahn EH, Voutilainen M, Fombonne J, Guix C, Viljakainen T, et al. The pair netrin-1/DCC regulates dopamine neuronal cell survival, death and impacts on Parkinson's disease. EMBO J. 2020 In Press.

63. Meng Z, Moroishi T, Guan KL. Mechanisms of Hippo pathway regulation. Genes Dev 2016;30:1-17.

64. Pellettieri J, Sanchez Alvarado A. Cell turnover and adult tissue homeostasis: from humans to planarians. Annu Rev Genet 2007;41:83-105.

65. Zhao B, Ye X, Yu J, Li L, Li W, Li S, et al. TEAD mediates YAP-dependent gene induction and growth control. Genes Dev 2008;22:1962-71.

66. Dong J, Feldmann G, Huang J, Wu S, Zhang N, Comerford $\mathrm{SA}$, et al. Elucidation of a universal size-control mechanism in Drosophila and mammals. Cell 2007;130:1120-33.

67. Lehtinen MK, Yuan Z, Boag PR, Yang Y, Villen J, Becker EB, et al. A conserved MST-FOXO signaling pathway mediates oxidative-stress responses and extends life span. Cell 2006; 125:987-1001.

68. Zhang P, Wang T, Zhang D, Zhang Z, Yuan S, Zhang J, et al. Exploration of MST1-mediated secondary brain injury induced by intracerebral hemorrhage in rats via hippo signaling pathway. Transl Stroke Res 2019;10:729-43.

69. Jang SW, Yang SJ, Srinivasan S, Ye K. Akt phosphorylates Mstl and prevents its proteolytic activation, blocking FOXO3 phosphorylation and nuclear translocation. J Biol Chem 2007;282:30836-44.

70. Ye K, Snyder SH. PIKE GTPase: a novel mediator of phosphoinositide signaling. J Cell Sci 2004;117(Pt 2):155-61.

71. Tang X, Jang SW, Okada M, Chan CB, Feng Y, Liu Y, et al. Netrin-1 mediates neuronal survival through PIKE-L interaction with the dependence receptor UNC5B. Nat Cell
Biol 2008;10:698-706.

72. Geng J, Sun X, Wang P, Zhang S, Wang X, Wu H, et al. Kinases Mst1 and Mst2 positively regulate phagocytic induction of reactive oxygen species and bactericidal activity. Nat Immunol 2015;16:1142-52.

73. Zhao S, Yin J, Zhou L, Yan F, He Q, Huang L, et al. Hippo/ MST1 signaling mediates microglial activation following acute cerebral ischemia-reperfusion injury. Brain Behav Immun 2016;55:236-48.

74. Shott RH, Majer A, Frost KL, Booth SA, Schang LM. Activation of pro-survival CaMK4 $\beta / C R E B$ and pro-death MST1 signaling at early and late times during a mouse model of prion disease. Virol J 2014;11:160.

75. Hoshino M, Qi ML, Yoshimura N, Miyashita T, Tagawa K, Wada Y, et al. Transcriptional repression induces a slowly progressive atypical neuronal death associated with changes of YAP isoforms and p73. J Cell Biol 2006;172:589-604.

76. Qi Q, Li DY, Luo HR, Guan KL, Ye K. Netrin-1 exerts oncogenic activities through enhancing Yes-associated protein stability. Proc Natl Acad Sci U S A 2015;112:7255-60.

77. Zhang Z, Kang SS, Liu X, Ahn EH, Zhang Z, He L, et al. Asparagine endopeptidase cleaves $\alpha$-synuclein and mediates pathologic activities in Parkinson's disease. Nat Struct Mol Biol 2017;24:632-42.

78. Bernet A, Mazelin L, Coissieux MM, Gadot N, Ackerman SL, Scoazec JY, et al. Inactivation of the UNC5C Netrin-1 receptor is associated with tumor progression in colorectal malignancies. Gastroenterology 2007;133:1840-8.

79. Thiebault K, Mazelin L, Pays L, Llambi F, Joly MO, Scoazec JY, et al. The netrin- 1 receptors UNC5H are putative tumor suppressors controlling cell death commitment. Proc Natl Acad Sci U S A 2003;100:4173-8.

80. Carvalho AL, Chuang A, Jiang WW, Lee J, Begum S, Poeta $L$, et al. Deleted in colorectal cancer is a putative conditional tumor-suppressor gene inactivated by promoter hypermethylation in head and neck squamous cell carcinoma. Cancer Res 2006;66:9401-7.

81. Feinstein E, Kimchi A, Wallach D, Boldin M, Varfolomeev E. The death domain: a module shared by proteins with diverse cellular functions. Trends Biochem Sci 1995;20:3424.

82. Park HH, Lo YC, Lin SC, Wang L, Yang JK, Wu H. The death domain superfamily in intracellular signaling of apoptosis and inflammation. Annu Rev Immunol 2007;25:561-86.

83. Dillon AK, Jevince AR, Hinck L, Ackerman SL, Lu X, Tessier-Lavigne $\mathrm{M}$, et al. UNC5C is required for spinal accessory motor neuron development. Mol Cell Neurosci 2007;35: 
482-9.

84. Leonardo ED, Hinck L, Masu M, Keino-Masu K, Ackerman SL, Tessier-Lavigne M. Vertebrate homologues of C. elegans UNC-5 are candidate netrin receptors. Nature 1997; 386:833-8.

85. Ackerman SL, Knowles BB. Cloning and mapping of the UNC5C gene to human chromosome 4q21-q23. Genomics 1998;52:205-8.

86. Kim D, Ackerman SL. The UNC5C netrin receptor regulates dorsal guidance of mouse hindbrain axons. J Neurosci 2011; 31:2167-79.
87. Yang B, Peng G, Gao J. Expression of unc5 family genes in zebrafish brain during embryonic development. Gene Expr Patterns 2013;13:311-8.

88. Wetzel-Smith MK, Hunkapiller J, Bhangale TR, Srinivasan $\mathrm{K}$, Maloney JA, Atwal JK, et al. A rare mutation in UNC5C predisposes to late-onset Alzheimer's disease and increases neuronal cell death. Nat Med 2014;20:1452-7.

89. Kang SS, Ahn EH, Zhang Z, Liu X, Manfredsson FP, Sandoval IM, et al. a-Synuclein stimulation of monoamine oxidase- $B$ and legumain protease mediates the pathology of Parkinson's disease. EMBO J 2018;37:e98878. 